Y. Kitaoka

Nagoya Math. J.

Vol. 103 (1986), 149-160

\title{
LOCAL DENSITIES OF QUADRATIC FORMS AND FOURIER COEFFICIENTS OF EISENSTEIN SERIES
}

\section{YOSHIYUKI KITAOKA}

Local densities of quadratic forms are important invariants in the theory of quadratic forms and they appear in Fourier coefficients of Eisenstein series. But it is not easy to evaluate them. To study their properties, it is desirable to look for relations among them, and it is known that there are many relations [3], but they are not concise. We consider a different kind of relations here and improve a result of Zharkovskaja $[8,9]$ in the case of Eisenstein series as an application.

Let $p$ be a prime number and $Z_{p}$ the ring of $p$-adic integers. We define local densities as follows: Put

$$
H_{2 k}=\frac{1}{2}\left[\begin{array}{rr}
0 & 1_{k} \\
1_{k} & 0
\end{array}\right]\left(1_{k}=\text { identity matrix of degree } k\right) .
$$

For a half-integral regular matrix $T$ of degree $n(\leqq 2 k)$ we define $\alpha_{p}\left(T, H_{2 k}\right)$ by

$$
\lim _{t \rightarrow \infty}\left(p^{t}\right)^{n(n+1) / 2-2 k n} \#\left\{C \in M_{2 k, n}\left(Z_{p} / p^{t} Z_{p}\right) \mid p^{-t}\left(H_{2 k}[C]-T\right) \text { : half-integral }\right\} .
$$

By definition $T$ is half-integral if and only if $2 T$ is a symmetric and integral matrix whose diagonal entries are in $2 Z_{p}$.

Our aim is to prove

Theorem 1. Lel $T$ be a half-integral regular matrix of degree $n(\leqq 2 k)$. Then the formal power series

$$
\sum_{r \geq 0} \alpha_{p}\left(p^{r} T, H_{2 k}\right) x^{r}
$$

is a rational function in $x$ with denominator

$$
\prod_{0 \leqq j \leqq n}\left(1-p^{(n-\jmath)(n+j+1-2 k) / 2} x\right)
$$

Received May 13, 1985. 
whose numerator has degree at most $n$. If, in addition $p^{-1} T$ is not halfintegral, then the degree of the numerator does not exceed $n-1$.

THEOREM 2. Let $a_{k}(T)$ be the Fourier coefficient of Eisenstein series $\sum_{\{C, D\}} \mid C Z+D_{\left.\right|^{-k}}$ of degree $n$, weight $k(k \equiv 0 \bmod 2, k>n+1)$. Then the formal power series

$$
\sum_{r \geqq 0} a_{k}\left(p^{r} T\right) x^{r}
$$

is a rational function for any positive definite half-integral $T$ with denominator

$$
\prod_{\jmath \leqq j \leqq n}\left(1-p^{j k-j(j+1) / 2} x\right)
$$

and the degree of the numerator is at most $n$, and at most $n-1$ if, in addition $p^{-1} T$ is not half-integral.

Remark. It is known [8,9] that the (not necessarily reduced) denominator of the formal power series in Theorem 2 is given by

$$
(1-x) \sum_{1 \leqq i_{1}<\cdots, \cdots i_{r} \leqq n}\left(1-p^{\sum_{1 \leqq j \leqq r}\left(k-i_{j}\right)} x\right) .
$$

Lemma 1. For a half-integral matrix $T$ of degree $n$ we put

$$
b(s, T)=\sum_{R} \nu(R)^{-s} e(\sigma(T R)),
$$

where $R$ runs over symmetric matrices in $M_{n}\left(\boldsymbol{Q}_{p} / Z_{p}\right)$ and $\nu(R)$ is a power of $p$ equal to the product of denominators of elementary divisors of $R$, and $\sigma$ is the trace and $e(z)$ means $\exp (2 \pi i(z \bmod 1))$ for $z \in \boldsymbol{Q}_{p}$. If $T$ is regular and $k$ is a natural number with $k \geqq n / 2$, then we have $b(k, T)=\alpha_{p}\left(T, H_{2 k}\right)$.

Proof. Let $T$ be a half-integral regular matrix of degree $n$. It is known $[4,7]$ that $b(s, T)$ is absolutely convergent for $s>n+1$ and a polynomial in $p^{-s}$, and $b(k, T)=\alpha_{p}\left(-T, H_{2 k}\right)$ for a sufficiently large integer $k$. The property that $\alpha_{p}\left(-T, H_{2 k}\right)$ is a polynomial comes from Lemmas 8,9 in [4]. Since Lemma 9 is valid for any integer $k \geqq n / 2$, the polynomial in $p^{-s}$ given by Lemmas 8,9 which is equal to $\alpha_{p}\left(-T, H_{2 k}\right)$ for a sufficiently large integer $k$ gives also $\alpha_{p}\left(-T, H_{2 k}\right)$ for any integer $k \geqq n / 2$. Thus we have $b(k, T)=b(k,-T)=\alpha_{p}\left(T, H_{2 k}\right)$ for an integer $k \geqq n / 2$. $N_{2}$ in Lemma 9 and Theorem 2 in [4] should be a maximal subspace which is totally singular and splits $N$. 
Lemma 2. For a half-integral matrix $T$ of degree $n$ and $s>n+1$, we have

$$
\prod_{0 \leqq i \leqq n-1}\left(1-p^{i-s}\right)^{-1} b(s, T)=\sum_{\lambda \in A} j(\lambda, T) p^{\sum_{1 \leqq i \leqq n} \lambda_{i}(n+1-i-s)} .
$$

where $\Lambda=\left\{\lambda=\left(\lambda_{1}, \cdots, \lambda_{n}\right) \mid 0 \leqq \lambda_{1} \leqq \cdots \leqq \lambda_{n}, \lambda_{i} \in Z\right\}$ and

$$
j(\lambda, T)=\#\left\{U \in \mathfrak{H}(\lambda) \backslash \mathfrak{H} \mid \begin{array}{l}
\text { For }\left(t_{i j}\right)=T\left[U^{-1}\right], \\
p^{\lambda_{i}}\left|t_{i i}, p^{\lambda_{i}}\right| 2 t_{i j} \quad \text { for } \quad i<j
\end{array}\right\} .
$$

Here we put $\mathfrak{U}=G L_{n}\left(Z_{p}\right)$ and $\mathfrak{H}(\lambda)=\mathfrak{H} \cap \chi(\lambda)^{-1} \mathfrak{H} \chi(\lambda)\left(\chi(\lambda)=\operatorname{diag}\left(p^{\lambda_{1}}, \cdots, p^{\lambda_{n}}\right)\right)$.

Proof. This is nothing but (2.8) in [2].

We put, for $0 \leqq k \leqq h \leqq n$

$$
\begin{gathered}
\Lambda_{k}=\left\{\lambda=\left(\lambda_{1}, \cdots, \lambda_{n}\right) \in \Lambda \mid \begin{array}{l}
\lambda_{i}=0 \text { if } i \leqq k, \\
\lambda_{i}>0 \text { if } i>k
\end{array}\right\}, \\
\Lambda_{k, h}=\left\{\lambda \in \Lambda_{k} \mid \lambda_{i}=1 \text { if } k<i \leqq h, \lambda_{i} \geqq 2 \text { if } i>h\right\} .
\end{gathered}
$$

Then it is easy to see

$$
\Lambda=\bigcup_{0 \leqq k \leqq n} \Lambda_{k} \text { (disjoint), } \Lambda_{k}=\bigcup_{k \leqq n \leqq n} \Lambda_{k, h} \text { (disjoint). }
$$

For $\lambda=\left(\lambda_{1}, \cdots, \lambda_{n}\right) \in \Lambda$ we define $\lambda-1$ by $\left(\mu_{1}, \cdots, \mu_{n}\right)$ with $\mu_{i}=\max \left(0, \lambda_{i}-1\right)$. Then the mapping $\lambda \rightarrow \lambda-1$ is clearly bijective from $\Lambda_{k, h}$ on $\Lambda_{h}$.

LEmma 3. Let $\lambda \in \Lambda_{k, h}$. Then $\mathfrak{H}(\lambda-1) \supset \mathfrak{H}(\lambda)$ and $[\mathfrak{H}(\lambda-1): \mathfrak{H}(\lambda)]=$ $p^{k(n-k)} \prod_{k+1 \leqq i \leqq h}\left(p^{-i}-1\right) \prod_{1 \leqq i \leqq h-k}\left(p^{-i}-1\right)^{-1}$ hold. Here $\prod_{\phi}$ means 1 .

Proof. Let $U=\left(u_{i j}\right) \in \mathfrak{H}$. $U \in \mathfrak{H}(\lambda)$ if and only if $p^{\lambda_{j}-\lambda_{i}} \mid u_{i j}$ for $i<j$. Hence we have only to prove $\mu_{j}-\mu_{i} \leqq \lambda_{j}-\lambda_{i}$ for $i<j, \mu=\left(\mu_{1}, \cdots, \mu_{n}\right)$ $=\lambda-1$ to show $\mathfrak{H}(\lambda-1) \supset \mathfrak{H}(\lambda)$. For $i<j$, we have

$$
\begin{aligned}
\lambda_{j}-\lambda_{i}-\left(\mu_{j}-\mu_{i}\right) & = \begin{cases}\lambda_{j}-\lambda_{i}-\left(\lambda_{j}-1\right)+\left(\lambda_{i}-1\right) & \text { if } i \geqq k+1, \\
\lambda_{j}-\lambda_{i}-\left(\lambda_{j}-1\right)+\lambda_{i} & \text { if } i \leqq k<j \\
\lambda_{j}-\lambda_{i}-\lambda_{j}+\lambda_{i} & \text { if } j \leqq k\end{cases} \\
& \geqq 0
\end{aligned}
$$

Thus we have $\mathfrak{H}(\lambda-1) \supset \mathfrak{H}(\lambda)$. By virtue of Lemma 6 in [1] we know

$$
[\mathfrak{H}: \mathfrak{H}(\lambda)]=p^{-\sum_{1 \leqq i \leqq n} \lambda_{i}(n-2 i+1)} \varphi_{n}\left(p^{-1}\right)\left(\varphi_{k_{1}}\left(p^{-1}\right) \cdots \varphi_{k_{t}}\left(p^{-1}\right)\right)^{-1},
$$

where $\varphi_{r}\left(p^{-1}\right)=\prod_{1 \leqq i \leqq r}\left(p^{-i}-1\right)$ and $\lambda=(\underbrace{\lambda_{1}^{\prime}, \cdots, \lambda_{1}^{\prime}}_{k_{1}}, \cdots, \underbrace{\lambda_{t}^{\prime}, \cdots, \lambda_{t}^{\prime}}_{k_{t}})$ with $\lambda_{1}^{\prime}$ 
$<\lambda_{2}^{\prime}<\cdots<\lambda_{\iota}^{\prime}$. Hence we have, for $\lambda \in \Lambda_{k, h}$

$$
\begin{aligned}
{[\mathfrak{H}(\lambda-1): \mathfrak{H}(\lambda)]=[\mathfrak{H}: \mathfrak{H}(\lambda)] /[\mathfrak{H}: \mathfrak{H}(\lambda-1)] } \\
\quad=p^{-\sum_{k<i \leqq n}(n-2 i+1)} \varphi_{h}\left(p^{-1}\right) \varphi_{k}\left(p^{-1}\right)^{-1} \varphi_{h-k}\left(p^{-1}\right)^{-1} \\
\quad=p^{-k(k-n)} \prod_{k+1 \leqq i \leqq h}\left(p^{-i}-1\right) \prod_{1 \leqq i \leqq h-k}\left(p^{-i}-1\right)^{-1} .
\end{aligned}
$$

Lemma 4. $j(\lambda, p T)=[\mathfrak{H}(\lambda-1): \mathfrak{H}(\lambda)] j(\lambda-1, T)$ holds for a half-integral matrix $T$ and $\lambda \in \Lambda$.

Proof. Put $\mu=\lambda-1$. Then we have

$[\mathfrak{H}(\mu): \mathfrak{H}(\lambda)] j(\mu, T)$

$$
\begin{aligned}
& =[\mathfrak{H}(\mu): \mathfrak{H}(\lambda)] \#\left\{\begin{array}{l}
U \in \mathfrak{H}(\mu)|\mathfrak{H}| \begin{array}{l}
\text { For }\left(t_{t_{i}}\right)=T\left[U^{-1}\right], \\
p^{\mu_{i}}\left|t_{i i}, p^{\mu_{i}}\right| 2 t_{i j}
\end{array} \text { for } i<j
\end{array}\right\} \\
& =\sharp\left\{U \in \mathfrak{H}(\lambda)|\mathfrak{U}| \text { For }\left(t_{i j}\right)=T\left[U^{-1}\right], p^{\mu_{i}}\left|t_{i i}, p^{\mu_{i}}\right| 2 t_{i j} \text { for } i<j\right\} \text {. }
\end{aligned}
$$

Since $\mu_{i}=0$ or $\lambda_{i}-1$ according to $\lambda_{i}=0$ or $\lambda_{i} \geqq 1$, the conditions $p^{\mu_{i}} t_{i i}$, $p^{\mu_{i}} \mid 2 t_{i j}$ are equal to $p^{\lambda_{i}}\left|p t_{i i}, p^{\lambda_{i}}\right| 2 p t_{i j}$ for $i<j$. Thus we complete the proof.

Combining Lemmas 3,4 we have

Lemma 5. If $\lambda \in \Lambda_{k, h}$, then we have

$$
j(\lambda, p T)=c(k, h) j(\lambda-1, T),
$$

where $c(k, h)=p^{k(n-k)} \prod_{k+1 \leqq i \leqq h}\left(p^{-i}-1\right) \prod_{1 \leqq i \leqq h-k}\left(p^{-i}-1\right)^{-1}$.

For a half-integral matrix $T$ of degree $n$ we denote by $f(x ; k, h)=$ $f(x ; k, h ; T)(0 \leqq k \leqq h \leqq n)$ a formal power series

$$
\sum_{r \geqq 0}\left(\sum_{\lambda \in \lambda_{k}, h} p^{\sum_{1 \leqq i \leqq n} \lambda_{i}(n+1-i-s)} j\left(\lambda, p^{r} T\right)\right) x^{r} .
$$

Coefficients of $x^{r}$ are absolutely convergent if $s>n+1$ by Lemma 2. We put

$$
\begin{aligned}
f(x) & =f(x ; T)=\sum_{0 \leqq k \leqq h \leqq n} f(x ; k, h) \\
& =\prod_{0 \leq i \leqq n-1}\left(1-p^{i-s}\right)^{-1} \sum_{r \geqq 0} b\left(s, p^{r} T\right) x^{r} .
\end{aligned}
$$

Since $b\left(s, p^{r} T\right)$ is rational in $p^{-s}$ [4], we have only to prove the assertion for $f(x)$ similar to Theorem 1 instead of $\sum_{r \geqq 0} \alpha_{p}\left(p^{r} T, H_{2 k}\right) x^{r}$ by virtue of Lemma 1.

Lemma 6. For $0 \leqq k \leqq h \leqq n$ we have 


$$
\begin{aligned}
f(x ; k, h)= & \sum_{\lambda \in \Lambda_{k}, h} p^{\sum_{1 \leqq i \leqq n} \lambda_{i}(n+1-i-s)} j(\lambda, T) \\
& +c(k, h) p^{(n-k)(n+1-k-2 s) / 2} \sum_{h \leqq f \leqq n} f(x ; h, f) x .
\end{aligned}
$$

Proof. By definition,

$$
\begin{aligned}
f(x ; k, h)= & \sum_{r \geqq 0} \sum_{\lambda \in \Lambda_{k}, h} p^{\sum \lambda_{i}(n+1-i-s)} j\left(\lambda, p^{r} T\right) x^{r} \\
= & \sum_{\lambda \in \Lambda_{k}, h} p^{\sum \lambda_{i}(n+1-i-s)} j(\lambda, T) \\
& +\sum_{r \geqq 0} \sum_{\lambda \in \Lambda_{k}, h} p^{\sum \lambda_{i}(n+1-i-s)} j\left(\lambda, p^{r+1} T\right) x^{r+1} \\
= & \sum_{\lambda \in \Lambda_{k}, h} p^{\sum \lambda_{i}(n+1-i-s)} j(\lambda, T) \\
& +c(k, h) \sum_{r \geqq 0} \sum_{\lambda \in \Lambda_{k}, h} p^{\sum \lambda_{i}(n+1-i-s)} j\left(\lambda-1, p^{r} T\right) x^{r+1}
\end{aligned}
$$

Since the mapping $\lambda \rightarrow \lambda-1$ is bijective from $\Lambda_{k, h}$ to $\Lambda_{h}$ and $\sum \lambda_{i}(n+1$ $-i-s)=\sum_{k<i \leqq n}(n+1-i-s)+\sum \mu_{i}(n+1-i-s)(\mu=\lambda-1)$, we complete the proof.

LEMma 7. For $0 \leqq a \leqq n$, we have

$$
\begin{aligned}
& \prod_{0 \leqq j \leqq a}\left(1-p^{(n-j)(n+j+1-2 s) / 2} x\right) f(x)=(\text { polynomial in } x \text { of degree } a-1)+\kappa(a) x^{a} \\
& \quad+x^{a+1} \sum_{a+1 \leqq k \leqq h \leqq n} A(a, k) f(x ; k, h)
\end{aligned}
$$

where $A(a, k)$ is independent of $h$ and $\kappa(a)$ satisfies

$$
\begin{aligned}
& \kappa(a+1)= \sum_{a+1 \leqq k \leqq h \leqq n} A(a, k) \sum_{\lambda \in \Lambda_{k}, h} p^{\sum \lambda_{i}(n+1-i-s)} j(\lambda, T) \\
&-p^{(n-a-1)(n+2+a-2 s) / 2} \kappa(a), \\
& \kappa(0)=f(0) .
\end{aligned}
$$

Proof. We use the induction on $a$.

$$
\begin{aligned}
(1 & \left.-p^{n(n+1-2 s) / 2} x\right) f(x) \\
& =f(x)-p^{n(n+1-2 s) / 2} x f(x) \\
& =f(0)+x \sum_{0 \leqq k \leqq h \leqq n}\left\{c(k, h) p^{(n-k)(n+1-k-2 s) / 2} \sum_{h \leqq f \leqq n} f(x ; h, f)-p^{n(n+1-2 s) / 2} f(x ; k, h)\right\} \\
& =f(0)+x \sum_{0 \leqq k \leqq h \leqq n} f(x ; k, h)\left\{\sum_{0 \leqq f \leqq k} c(f, k) p^{(n-f)(n+1-f-2 s) / 2}-p^{n(n+1-2 s) / 2}\right\} \\
& =f(0)+x \sum_{1 \leqq k \leqq h \leqq n} f(x ; k, h)\left\{\sum_{1 \leqq f \leqq k} c(f, k) p^{(n-f)(n+1-f-2 s) / 2}\right\} .
\end{aligned}
$$

For $A(0, k)=\sum_{1 \leqq f \leqq k} c(f, k) p^{(n-f)(n+1-f-2 s) / 2}$, the first step has been proved. For $0 \leqq a \leqq n-1$, 


$$
\begin{aligned}
& \prod_{0 \leqq j \leqq a+1}\left(1-p^{(n-j)(n+j+1-2 s) / 2} x\right) f(x) \\
& =\left(1-p^{(n-a-1)(n+2+a-2 s) / 2} x\right)\{(\text { polynomial in } x \text { of degree } a-1) \\
& \left.+\kappa(a) x^{a}+x^{a+1} \sum_{a+1 \leqq k \leqq h \leqq n} A(a, k) f(x ; k, h)\right\} \\
& =(\text { polynomial in } x \text { of degree } a) \\
& +x^{a+1}\left\{\sum_{a+1 \leqq k \leqq h \leqq n} A(a, k) f(x ; k, h)-\kappa(a) p^{(n-a-1)(n+2+a-2 s) / 2}\right\} \\
& -x^{a+2} p^{(n-a-1)(n+2+a-2 s) / 2} \sum_{a+1 \leqq k \leqq h \leqq n} A(a, k) f(x ; k, h) \text {. }
\end{aligned}
$$

By Lemma 6, we have

$$
\begin{aligned}
\sum_{a+1 \leqq k \leqq h \leqq n} A(a, k) f(x ; k, h) & \\
= & \sum_{a+1 \leqq k \leqq h \leqq n} A(a, k) \sum_{\lambda \in \Lambda_{k}, h} p^{\sum^{\lambda_{i}(n+1-i-s)} j(\lambda, T)} \\
& +\sum_{a+1 \leqq k \leqq h \leqq n} A(a, k) c(k, h) p^{(n-k)(n+1-k-2 s) / 2} \sum_{h \leqq f \leqq n} f(x ; h, f) x .
\end{aligned}
$$

Thus we have

$$
\begin{aligned}
\prod_{0 \leqq j \leqq a+1} & \left.\left(1-p^{(n-j)(n+1+j-2 s) / 2} x\right) f(x)=\text { (polynomial in } x \text { of degree } a\right) \\
& +x^{a+1}\left\{\sum_{a+1 \leqq k \leqq h \leqq n} A(a, k) \sum_{\lambda \in \Lambda_{k}, h} p^{\sum \lambda_{i}(n+1-i-s)} j(\lambda, T)-\kappa(a) p^{(n-a-1)(n+2+a-2 s) / 2}\right\} \\
& +x^{a+2} \sum_{a+1 \leqq k \leqq h \leqq n}\left\{A(a, k) c(k, h) p^{(n-k)(n+1-k-2 s) / 2} \cdot \sum_{h \leqq f \leqq n} f(x ; h, f)\right. \\
& \left.-p^{(n-a-1)(n+2+a-2 s) / 2} A(a, k) f(x ; k, h)\right\} .
\end{aligned}
$$

The last term is equal to

$$
\begin{aligned}
x^{a+2} & \sum_{a+1 \leqq k \leqq h \leqq n} f(x ; k, h)\left\{\sum_{a+1 \leqq f \leqq k} A(a, f) c(f, k) p^{(n-f)(n+1-f-2 s) / 2}\right. \\
& \left.-p^{(n-a-1)(n+2+a-2 s) / 2} A(a, k)\right\} \\
= & x^{a+2} \sum_{a+2 \leqq k \leqq h \leqq n} f(x ; k, h)\left\{\sum_{a+1 \leqq f \leqq k} A(a, f) c(f, k) p^{(n-f)(n+1-f-2 s) / 2}\right. \\
& \left.-p^{(n-a-1)(n+2+a-2 s) / 2} A(a, k)\right\} .
\end{aligned}
$$

Putting

$$
A(a+1, k)=\sum_{a+1 \leqq f \leqq k} A(a, f) c(f, k) p^{(n-f)(n+1-f-2 s) / 2}-p^{(n-a-1)(n+2+a-2 s) / 2} A(a, k),
$$

we complete the proof.

From Lemma 7 follows that $\prod_{0 \leqq j \leqq n}\left(1-p^{(n-j)(n+j+1-2 s) / 2} x\right) f(x)$ is a polynomial of degree $n$ whose leading coefficient is $k(n)$. It remains for us to prove Theorem 1 that $\kappa(n)=0$ if $p^{-1} T$ is not half-integral.

Lemma 8. Put $g(f)=p^{(n-f)(n+f+1-2 s) / 2}$, 


$$
D(f, k)=\prod_{f+1 \leqq i \leqq k}\left(p^{-i}-1\right) \prod_{1 \leqq i \leqq k-f}\left(p^{-i}-1\right)^{-1} .
$$

Then we have

$$
\begin{aligned}
& A(0, k)=\sum_{1 \leqq f \leqq k} g(f) D(f, k), \\
& A(a+1, k)=\sum_{a+1 \leqq f \leqq k} A(a, f) g(f) D(f, k)-g(a+1) A(a, k) \\
& \kappa(n)=\sum_{0 \leqq k \leqq h \leqq n} J(k, h)\left\{\sum_{0 \leqq i \leqq k-1}\left(\prod_{i+2 \leqq j \leqq n}(-g(j))\right) A(i, k)+\prod_{1 \leqq j \leqq n}(-g(j))\right\},
\end{aligned}
$$

where

$$
J(k, h)=\sum_{\lambda \in \Lambda_{k}, h} p^{\sum \lambda_{i}(n+1-i-s)} j(\lambda, T) .
$$

Proof. The first two are nothing but their definition. To prove the last we show the following inductively:

$$
\begin{aligned}
\kappa(a)= & \sum_{0 \leqq i \leqq a-1}\left(\prod_{i+2 \leqq j \leqq a}(-g(j))\right) \sum_{i+1 \leqq k \leqq h \leqq n} A(i, k) J(k, h) \\
& +\prod_{1 \leqq i \leqq a}(-g(i)) \sum_{0 \leqq k \leqq h \leqq n} J(k, h) .
\end{aligned}
$$

Here we put $\sum_{\phi}=0$. Then it is clearly true for $a=0$. Let $0 \leqq a \leqq n-1$; then by Lemma 7

$$
\begin{aligned}
\kappa(a+1)= & \sum_{a+1 \leqq k \leqq h \leqq n} A(a, k) J(k, h)-g(a+1) \kappa(a) \\
= & \sum_{a+1 \leqq k \leqq h \leqq n} A(a, k) J(k, h) \\
& -g(a+1)\left\{\sum_{0 \leqq i \leqq a-1}\left(\prod_{i+2 \leqq j \leqq a}(-g(j))\right) \sum_{i+1 \leqq k \leqq h \leqq n} A(i, k) J(k, h)\right. \\
& \left.+\prod_{1 \leqq i \leqq a}(-g(i)) \sum_{0 \leqq k \leqq h \leqq n} J(k, h)\right\} \\
= & \sum_{0 \leqq i \leqq a}\left(\prod_{i+2 \leqq j \leqq a+1}(-g(j))\right) \sum_{i+1 \leqq k \leqq h \leqq n} A(i, k) J(k, h) \\
& +\left(\prod_{1 \leqq i \leqq a+1}(-g(i))\right) \sum_{0 \leqq k \leqq h \leqq n} J(k, h) .
\end{aligned}
$$

Applying it to $a=n$, we prove Lemma 8 .

If $p^{-1} T$ is not half-integral, then $J(0, h)=0$ follows from $j(\lambda, T)=0$ for $\lambda \in \Lambda_{0, h}$ since $\lambda_{1} \geqq 1$ for $\lambda \in \Lambda_{0, h}$. Hence, to complete the proof of Theorem 1 , we have only to prove

LEMma $\left.9 . \quad \kappa(n)=\sum_{0 \leqq h \leqq n} J(0, h) \prod_{1 \leqq j \leqq n}(-g(j))\right)$, more minutely

$$
\sum_{0 \leqq i \leqq k-1}\left(\prod_{i+2 \leqq j \leqq n}(-g(j))\right) A(i, k)+\prod_{1 \leqq j \leqq n}(-g(j))=0 \quad \text { for } 1 \leqq k \leqq n .
$$

This follows directly from the following 
Lemma 10. Let $D(a, b), g(a)$ be independent variables for $1 \leqq a<b$ and put $D(a, a)=1$ for $a \geqq 1$. Define $A(a, b)$ for $0 \leqq a<b$ inductively by

$$
\begin{gathered}
A(0, m)=\sum_{1 \leqq f \leqq m} g(f) D(f, m), \\
A(a+1, m)=\sum_{a+1 \leqq f \leqq m} A(a, f) g(f) D(f, m)-g(a+1) A(a, m) \\
\quad \text { for } 0 \leqq a<m-1 .
\end{gathered}
$$

Then we have, for $0 \leqq k \leqq m-1$

$$
F(k, m)=\sum_{0 \leqq i \leqq k}\left(\prod_{i+2 \leqq j \leqq m}(-g(j))\right) A(i, k+1)+\prod_{1 \leqq j \leqq m}(-g(j))=0 .
$$

Proof. We use the induction on $m$. Since $F(0,1)=A(0,1)-g(1)=0$, the first step is true. Suppose $m \geqq 2$ and $F(k, n)=0$ for $0 \leqq k \leqq n-1 \leqq$ $m-2$. If $k \leqq m-2$, then we have

$$
F(k, m)=-g(m) F(k, m-1)=0 .
$$

It remains for us to prove $F(m-1, m)=0$ for $m \geqq 2$. We put

$$
\begin{aligned}
F(m) & =F(m-1, m) \\
& =\sum_{0 \leqq i \leqq m-2}\left(\sum_{i+2 \leqq j \leqq m}(-g(j))\right) A(i, m)+A(m-1, m)+\prod_{1 \leqq j \leqq m}(-g(j)) .
\end{aligned}
$$

We prove $F(m)=0$ by showing that all coefficients of the polynomial $F(m)$ in $g(m)$ vanish.

Sublemma 1. $A(a, b)$ does not contain $g(m)$ for $b<m$, and $A(a, m)$ is a monic polynomial in $g(m)$ of degree $a+1$, and $F(m)$ is of degree at most $m$.

Proof. The first assertion is easily proved by the induction on $a$. The second is also proved by the induction on $a: A(0, m)=\sum_{1 \leqq f \leqq m} g(f) D(f, m)$ $=\sum_{1 \leqq f \leqq m-1} g(f) D(f, m)+g(m)$ is a monic polynomial of degree one. For $0 \leqq a \leqq m-2$, we have

$$
\begin{aligned}
A(a+1, m)= & \sum_{a+1 \leqq f \leqq m} A(a, f) g(f) D(f, m)-g(a+1) A(a, m) \\
= & \sum_{a+1 \leqq f \leqq m-1} A(a, f) g(f) D(f, m)+A(a, m) g(m) \\
& -g(a+1) A(a, m) .
\end{aligned}
$$

By the inductive assumption and the first assertion, $A(a+1, m)$ is a monic polynomial in $g(m)$ of degree $a+2$. Now the last assertion is clear.

Sublemma 2. For $2 \leqq q \leqq m$, the coefficient of $g(m)^{q}$ of the polynomial 
$F(m)$ in $g(m)$ vanishes.

Proof. Write $A(a, m)=\sum_{0 \leqq i \leqq a+1} h(i ; a, m) g(m)^{i}$, where $h(i ; a, m)$ does not contain $g(m)$. Then we have

$$
\begin{aligned}
& h(a+1 ; a, m)=1 \text { and } \\
& h(i ; a+1, m)=h(i-1 ; a, m)-g(a+1) h(i ; a, m) \quad \text { for } i \geqq 1,
\end{aligned}
$$

by the definition of $A(a, m)$ and the above sublemma. We complete the proof of this sublemma by the induction on $q=m, m-1, \cdots, 2$. By Sublemma 1 , the coefficient of $g(m)^{m}$ is 0 . Suppose that the coefficient of $g(m)^{r}$ vanishes for $r=q+1, \cdots, m(2 \leqq q \leqq m-1)$. The coefficient of $g(m)^{q}$ is

$$
\begin{aligned}
& -\sum_{\substack{0 \leqq i \leqq m-2 \\
q-1 \leqq i+1}} \prod_{i+2 \leqq j \leqq m-1}(-g(j)) h(q-1 ; i, m)+h(q ; m-1, m) \\
& =-\prod_{q \leqq j \leqq m-1}(-g(j))-\sum_{q-1 \leqq i \leqq m-2}\left(\prod_{i+2 \leqq j \leqq m-1}(-g(j))\right) h(q-1 ; i, m) \\
& \quad+h(q ; m-1, m) .
\end{aligned}
$$

For $q-1 \leqq t \leqq m-2$, we have

$$
\begin{aligned}
h(q ; t+ & 1, m)-\sum_{q-1 \leqq i \leqq t}\left(\prod_{i+2 \leqq j \leqq t+1}(-g(j))\right) h(q-1 ; i, m)-\sum_{q \leqq j \leqq t+1}(-g(j)) \\
= & h(q-1 ; t, m)-g(t+1) h(q ; t, m)-h(q-1 ; t, m) \\
& -\sum_{q-1 \leqq i \leqq t-1}\left(\prod_{i+2 \leqq j \leqq t+1}(-g(j))\right) h(q-1 ; i, m)-\prod_{q \leqq j \leqq t+1}(-g(j)) \\
= & -g(t+1)\left\{h(q ; t, m)-\sum_{q-1 \leqq i \leqq t-1}\left(\prod_{i+2 \leqq j \leqq t}(-g(j))\right) h(q-1 ; i, m)\right. \\
& \left.-\prod_{q \leqq j \leqq t}(-g(j))\right\} .
\end{aligned}
$$

Applying this to the coefficient of $g(m)^{q}$ from $t=m-2$ to $t=q-1$, it is equal to

$$
(-g(m-1)) \cdots(-g(q)) 0=0 .
$$

Sublemma 3. The coefficient of $g(m)$ of the polynomial $F(m)$ vanishes.

Proof. The coefficient of $g(m)$ is equal to

$$
-\sum_{0 \leqq i \leqq m-2}\left(\prod_{i+2 \leqq j \leqq m-1}(-g(j))\right) h(0 ; i, m)+h(1 ; m-1, m)-\prod_{1 \leqq j \leqq m-1}(-g(j)) \text {. }
$$

We show inductively

$$
h(1 ; a, m)=\sum_{0 \leqq i \leqq a-1}\left(\prod_{i+2 \leqq j \leqq a}(-g(j))\right) h(0 ; i, m)+\prod_{1 \leqq j \leqq a}(-g(j)) .
$$

For $a=0$, both sides are equal to 1 . Suppose that the above formula is true for $a$. Then 


$$
\begin{aligned}
h(1 ; a & +1, m) \\
& =h(0 ; a, m)-g(a+1) h(1 ; a, m) \\
& =h(0 ; a, m)+\sum_{0 \leqq i \leqq a-1}\left(\prod_{i+2 \leqq j \leqq a+1}(-g(j))\right) h(0 ; i, m)+\prod_{1 \leqq j \leqq a+1}(-g(j)) \\
& =\sum_{0 \leqq i \leqq a}\left(\prod_{i+2 \leqq j \leqq a+1}(-g(j))\right) h(0 ; i, m)+\prod_{1 \leqq j \leqq a+1}(-g(j)) .
\end{aligned}
$$

Thus the above formula is proved, and the case of $a=m-1$ is what we want.

Thus it has been proved that $F(m)$ is a constant with respect to $g(m)$ and hence we have only to prove

$$
F(m)=h(0 ; m-1, m)=0 .
$$

Sublemma 4. For $0 \leqq a \leqq m-1$, we have

$$
h(0 ; a, m)=\left(\prod_{1 \leqq i \leqq a}(-g(i))\right) h(0 ; 0, m)+\sum_{0 \leqq j \leqq a-1}\left(\prod_{j+2 \leqq i \leqq a}(-g(i))\right) G(j),
$$

where $G(j)=\sum_{j+1 \leqq f \leqq m-1} A(j, f) g(f) D(f, m)$.

Proof. When $a=0$, both sides are equal to $h(0 ; 0, m)$. By definition. we have, for $0 \leqq a \leqq m-2$

$$
\begin{aligned}
h(0 ; a+1, m)= & \sum_{a+1 \leqq f \leqq m-1} A(a, f) g(f) D(f, m)-g(a+1) h(0 ; a, m) \\
= & G(a)-g(a+1) h(0 ; a, m) \\
= & G(a)+\left(\prod_{1 \leqq i \leqq a+1}(-g(i))\right) h(0 ; 0, m) \\
& +\sum_{0 \leqq j \leqq a-1}\left(\prod_{j+2 \leqq i \leqq a+1}(-g(i))\right) G(j) \\
= & \left(\prod_{1 \leqq i \leqq a+1}(-g(i))\right) h(0 ; 0, m)+\sum_{0 \leqq j \leqq a}\left(\prod_{j+2 \leqq i \leqq a+1}(-g(i))\right) G(j) .
\end{aligned}
$$

Sublemma 5. $h(0 ; m-1, m)=0$.

Proof. $\quad h(0 ; 0, m)=\sum_{1 \leqq f \leqq m-1} g(f) D(f, m)$ and $G(j)=\sum_{j+1 \leqq f \leqq m-1} A(j, f)$ $g(f) D(f, m)$ follow from their definition. By Sublemma 4 we have

$$
\begin{aligned}
h(0 ; m & -1, m) \\
= & \left(\prod_{1 \leqq i \leqq m-1}(-g(i))\right) \sum_{1 \leqq j \leqq m-1} g(f) D(f, m) \\
& +\sum_{0 \leqq j \leqq m-2}\left(\prod_{j+2 \leqq i \leqq m-1}(-g(i))\right) \sum_{j+1 \leqq f \leqq m-1} A(j, f) g(f) D(f, m) \\
= & \sum_{1 \leqq j \leqq m-1} g(f) D(f, m)\left\{\prod_{1 \leqq i \leqq m-1}(-g(i))+\sum_{0 \leqq j \leqq f-1}\left(\prod_{j+2 \leqq i \leqq m-1}(-g(i))\right) A(j, f)\right\} \\
= & \sum_{1 \leqq f \leqq m-1} g(f) D(f, m) \prod_{f+1 \leqq i \leqq m-1}(-g(i))\left\{\prod_{1 \leqq i \leqq f}(-g(i))\right. \\
& \left.+\sum_{0 \leqq j \leqq f-1}\left(\prod_{j+2 \leqq i \leqq f}(-g(i))\right) A(j, f)\right\}
\end{aligned}
$$




$$
=\sum_{1 \leqq f \leqq m-1} g(f) D(f, m)\left(\prod_{f+1 \leqq i \leqq m-1}(-g(i))\right) F(f)=0,
$$

since $F(f)=0$ for $1 \leqq f \leqq m-1$ by the inductive assumption.

Thus we have completed the proof of Theorem 1 .

Proof of Theorem 2. Let $T$ be a half-integral positive definite matrix of degree $n$. Then $a_{k}(T)$ is given in [6] by

$$
(-1)^{n k / 2} 2^{n(k-(n-1) / 2)} \prod_{0 \leqq i \leqq n-1} \frac{\pi^{k-i / 2}}{\Gamma(k-i / 2)}|T|^{k-(n+1) / 2} \cdot \prod_{p} \alpha_{p}\left(T, H_{2 k}\right) .
$$

For a unit $\varepsilon \in Z_{p}^{\times}$, we have $\alpha_{p}\left(T, H_{2 k}\right)=b(k, T)=b(k, \varepsilon T)=\alpha_{p}\left(\varepsilon T, H_{2 k}\right)$. Hence we have

$$
\sum_{r \geqq 0} a_{k}\left(p^{r} T\right) x^{r}=\text { const } \sum_{r \geqq 0} \alpha_{p}\left(p^{r} T, H_{2 k}\right)\left(p^{n k-(n+1) n / 2} x\right)^{r},
$$

which is a rational function in $x$ whose denominator is

$$
\begin{gathered}
\prod_{0 \leqq j \leqq n}\left(1-p^{(n-j)(n+1-2 k+j) / 2+n k-(n+1) n / 2} x\right) \\
=\prod_{0 \leqq j \leqq n}\left(1-p^{j k-j(j+1) / 2} x\right) .
\end{gathered}
$$

Another assertion follows from the corresponding one in Theorem 1.

Remark. The formal power series similar to one in Theorem 1 for any quadratic form instead of $H_{2 k}$ seems to be rational.

\section{REFERENCES}

[1] A. N. Andrianov, Spherical functions for $G L_{n}$ over local fields, and summation of Hecke series, Math. USSR Sbornik, 12 (1970), 429-452.

[2] G. Kaufhold, Dirichletsche Reihe mit Funktionalgleichung in der Theorie der Modulfunktion 2. Grades, Math. Ann., 137 (1959), 454-476.

[ 3 ] Y. Kitaoka, A note on local densities of quadratic forms, Nagoya Math. J., 92 (1983), 145-152.

[4] - Dirichlet series in the theory of Siegel modular forms, Nagoya Math. J., 95 (1984), 73-84.

[5] — Fourier coefficients of Eisenstein series of degree 3, Proc. Japan Acad., 60 (1984), 259-261.

[6] H. Maaß, Die Fourierkoeffizienten der Eisensteinreihen zweiten Grades, Mat.-Fys. Medd. Danske Vid. Selsk., 34 (1964), no. 7.

[ 7 ] C. L. Siegel, On the theory of indefinite quadratic forms, Ann. Math. 45 (1944), $577-622$.

[ 8 ] N. A. Zharkovskaja, The Siegel operator and Hecke operators, Functional anal. Appl., 8 (1974), 113-120.

[9] - On the connection of the eigenvalues of Hecke operators and the Fourier 
coefficients of eigenfunctions for Siegel's modular forms of genus $n$, Math. USSR Sbornik, 25 (1975), 549-557.

Department of Mathematics

Nagoya University

Chikusa-ku, Nagoya 464

Japan 